\section{Vítimas da cor: homicídios na região metropolitana de São Paulo, Brasil, 2000}

\author{
Victims of color: homicides in Greater \\ Metropolitan São Paulo, Brazil, 2000
}

Samuel Kilsztajn 1

Manuela Santos Nunes do Carmo ${ }^{1}$

Gustavo Toshiaki Lopes Sugahara 1

Erika de Souza Lopes ${ }^{1}$

\footnotetext{
1 Programa de Estudos

Pós-graduados em Economia

Politica, Pontifícia

Universidade Católica de

São Paulo, São Paulo, Brasil.

Correspondência

S. Kilsztajn

Laboratório de Economia

Social, Programa de

Estudos Pós-graduados

em Economia Política,

Pontifícia Universidade

Católica de São Paulo.

Rua Marquês de Paranaguá

164, apto. 602, São Paulo, SP

01303-050, Brasil.

skil@pucsp.br
}

\begin{abstract}
The objective of this paper was to analyze the homicide rate by racial/ethnic origin in Greater Metropolitan São Paulo, Brazil, after controlling for schooling, gender, and age. Based on available vital statistics for São Paulo and from the National Census, the homicide rates for blacks and non-blacks in Greater Metropolitan São Paulo in 2000 were calculated for 134 units (38 municipalities and 96 capital districts). In addition, two categories (reference and exposure) were created for each of the following death certificate socio-demographic data: race, schooling, gender, and age. The research used descriptive and logistic regression analyses. Although homicide rates were invariably higher for blacks than for non-blacks, the race variable was not statistically significant when schooling, gender, and age were controlled. The higher homicide rate for blacks in Greater Metropolitan São Paulo results from overrepresentation of blacks both among individuals with low schooling and in the young male population, the principal exposure categories for homicide.
\end{abstract}

Homicide; Mortality Rate; Cause of Death; Blacks

\section{Introdução}

Nas três últimas décadas, as causas externas de mortalidade, e os homicídios em particular, têm ocupado lugar de destaque no quadro nacional de saúde 1. Os Indicadores e Dados Básicos para a Saúde 2 registram uma taxa de mortalidade específica por homicídio de 27,8 por $100 \mathrm{mil}$ habitantes no Brasil em 2001, inferior somente à da Colômbia, 60,8 por 100 mil habitantes em 1995 3. Por 100 mil habitantes, os Estados Unidos registram uma taxa de homicídio de 6,6 e a Alemanha, Áustria, Espanha, França, Reino Unido, Austrália, China e Japão taxas inferiores a 1,0 (dados relativos a 1998 ou 1999) 3 .

No Brasil, os homicídios atingem principalmente as regiões metropolitanas, que apresentaram uma taxa de homicídio de 40,7 por 100 mil habitantes em 2001; e São Paulo, a maior área metropolitana brasileira, ostentava uma taxa de homicídio de 58,2 por 100 mil habitantes em 20012.

A evolução e os determinantes da taxa de homicídio têm sido abordados com destaque na literatura nacional e internacional em várias áreas do conhecimento 1,4,5,6. A relação entre mortalidade por homicídio e raça, especificamente, tem também constituído objeto de estudo de várias pesquisas 7,8,9,10.

No contexto das relações raciais, o Brasil é freqüentemente confundido com uma democracia racial porque o preconceito aqui apare- 
ce muitas vezes como um objeto invisível e o país não apresenta tensões abertas e conflitos permanentes 11 . Na aparente inconsistência das estatísticas, a quase totalidade dos brancos, quando entrevistada, afirma que não se julga racista; mas diz conhecer pessoas próximas que têm preconceitos. Da mesma forma, a maioria dos negros entrevistados nega ter sido vítima de discriminação; mas confirma casos de racismo envolvendo familiares e conhecidos próximos. Neste sentido, racista e vítima de racismo, no Brasil, é sempre o outro 12.

A questão negra não se resume à questão social. Os negros estão sobre-representados nas camadas pobres, de baixa escolaridade, no trabalho infantil, no trabalho informal, nos empregos domésticos (de lavadeira/passador, lixeiro, varredor etc.); por outro lado, estão excluídos ou sub-representados entre advogados, juízes, médicos, dentistas, engenheiros, professores universitários etc. 13. E o valor do rendimento pessoal do trabalho de negros é sempre inferior ao das demais categorias de raça/cor, mesmo quando se controla o nível de escolaridade 14,15 .

O presente estudo tem por objetivo analisar as taxas de homicídio por raça, controladas as variáveis escolaridade, sexo e idade da vítima em 2000, na Região Metropolitana de São Paulo, que possui uma identidade econômicosocial que se sobrepõe à divisão política da região em municípios.

\section{Métodos}

Até 1999, a Fundação Seade 16 registrava os homicídios ocorridos no Estado de São Paulo com município de residência da vítima ignorado como residentes no município de ocorrência. A partir de 2000, entretanto, a Fundação Seade passou a registrar esses homicídios como residentes no Estado de São Paulo com município de residência ignorado. O Programa de Aprimoramento de Dados sobre a Mortalidade no Município de São Paulo (PRO-AIM) 17, por sua vez, considerando que a maior parte das vítimas de homicídio reside no município de ocorrência, continua registrando os homicídios ocorridos na capital com município de residência da vítima ignorado como homicídios de residentes no Município de São Paulo. De acordo com a Fundação Seade 16, em 2000, 99,1\% dos homicídios de residentes na Região Metropolitana de São Paulo ocorreram na própria re- gião, onde 98,8\% das vítimas de homicídio com município de residência da vítima informado residiam na Região Metropolitana de São Paulo. Para a presente pesquisa, optou-se, portanto, por manter os homicídios ocorridos na Região Metropolitana de São Paulo com município de residência da vítima ignorado como homicídios de residentes na Região Metropolitana de São Paulo.

Com base nas estatísticas vitais da Fundação Seade 16 e nos microdados da amostra do Censo Demográfico do Instituto Brasileiro de Geografia e Estatística (IBGE) 18, foram calculadas as taxas de homicídio em 2000 para a raça negra e para os não-negros em 134 unidades da Região Metropolitana de São Paulo (38 municípios e a capital subdividida em 96 distritos).

Para a população, de acordo com o critério do IBGE, a raça/cor é autodenominada pelo entrevistado entre cinco categorias pré-codificadas (branca, preta, amarela, parda e indígena); para a vítima de homicídio, por sua vez, a raça/cor é atribuída pelo médico legista entre as cinco categorias acima citadas.

O termo negro foi utilizado para designar pessoas pretas e pardas. A análise conjunta de pretos e pardos é utilizada por Silva 15 , Henriques 13 e Jacooud \& Beghin 19, entre outros autores, porque o padrão de renda média por escolaridade, experiência e outras variáveis socioeconômicas é extraordinariamente semelhante entre pretos e pardos e substancialmente diferente entre estes e as demais categorias de raça/cor.

Para o agregado da Região Metropolitana de São Paulo, foram construídas duas categorias (de referência e de exposição) para cada uma das quatro variáveis sócio-demográficas da Declaração de Óbito: sexo, anos de estudo, idade e raça (dada a qualidade da informação, a variável ocupação não foi utilizada no modelo). As categorias consideradas de exposição foram sexo masculino, até sete anos de estudo (fundamental incompleto), de 15 a 34 anos de idade e negra (referência: sexo feminino, 8 e mais anos de estudo, 35 e mais anos de idade e não-negra).

Considerando que as pessoas até 14 anos de idade não tiveram tempo de concluir oito anos de estudo (que é o corte da categoria de referência para a variável anos de estudo), as taxas de homicídio para as quatro variáveis mencionadas foram calculados para a população com 15 e mais anos de idade. A informação sobre a escolaridade da vítima na Declaração 
de Óbito, desta forma, pode ser utilizada como uma aproximação do rendimento, na medida que escolaridade e rendimento no Brasil são altamente correlacionados 20. A exclusão de menores de 15 anos justifica-se também pela baixa taxa de homicídio dessa faixa etária 1 .

Para a análise do agregado da Região Metropolitana de São Paulo foram utilizadas apenas as vítimas de homicídio com 15 e mais anos de idade que apresentavam informações simultâneas para sexo, escolaridade, idade e raça/cor. Para a análise descritiva foram criados 16 grupos a partir das variáveis sexo, anos de estudo, idade e raça desdobradas nas categorias de referência e de exposição. Cada um desses 16 grupos, neste sentido, é homogêneo, isto é, composto por pessoas do mesmo sexo, escolaridade, faixa etária e identificação étnica.

Para a análise multivariada de regressão logística foram testados dois modelos: o primeiro com a utilização das variáveis raça e anos de estudo, e o segundo com a introdução das variáveis demográficas sexo e idade (considerado-se os homicídios e a população com 15 e mais anos de idade na Região Metropolitana de São Paulo em 2000).

A amostra do Censo Demográfico, de acordo com seu desenho, atribui diferentes pesos para as observações. Para garantir o tamanho da amostra e sua representatividade, concomitantemente, a amostra selecionada foi primeiramente expandida a partir do "peso da pessoa". O peso original de cada observação foi poste- riormente dividido pelo fator $v / \alpha$, em que $v$ é o tamanho do universo e $\alpha$ o tamanho da amostra (o número de homicídios, por conseguinte, também foi dividido pelo fator $v / \alpha$ ).

Especificamente para a raça, foi avaliada a distribuição da população por raça nas três demais variáveis analisadas.

\section{Resultados}

A Fundação Seade registrou 10.604 homicídios de residentes na Região Metropolitana de São Paulo e 685 homicídios ocorridos na Região Metropolitana de São Paulo com município de residência da vítima ignorado em 2000. Por 100 mil habitantes, considerando-se 11.289 homicídios $(10.604+685)$ e 17,9 milhões de habitantes, a taxa de homicídio atingiu 63,1 para a Região Metropolitana de São Paulo em 2000, 87,5 para negros e 51,5 para não-negros, com risco relativo de homicídio da população negra em relação à não-negra de 1,7 (a raça/cor não foi informada em 106 registros de homicídio).

A Figura 1 apresenta a taxa de homicídio por 100 mil habitantes para cada uma das 134 unidades da Região Metropolitana de São Paulo (38 municípios e a capital subdividida em 96 distritos) para o total da população, para negros e para não-negros. Para essas taxas puderam ser utilizados os 10.604 homicídios com município de residência da vítima informado, com exclusão de 257 casos (77 com raça/cor não

Figura 1

Taxa de homicídio por raça. Região Metropolitana de São Paulo, Brasil, 2000.

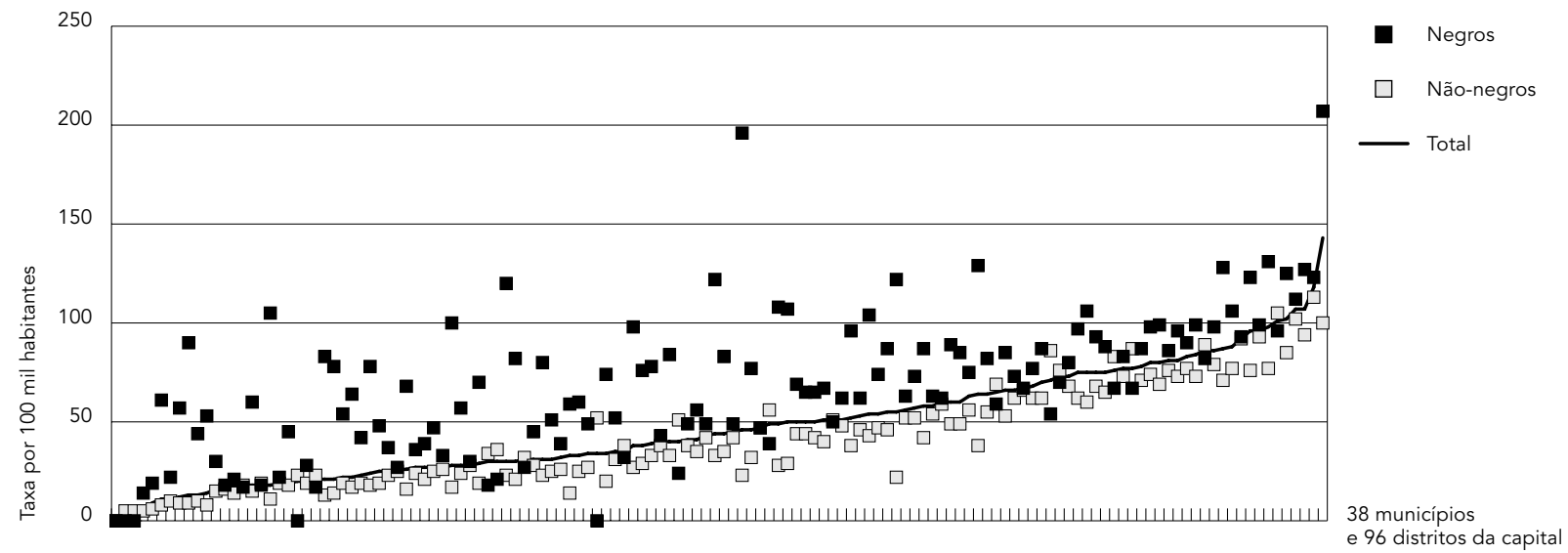

Fonte: Fundação Seade 16 e IBGE 18. 
informada e 180 com distrito de residência na capital não informado).

As 134 unidades geográficas foram alinhadas na Figura 1 em ordem crescente, de acordo com a taxa de homicídio dos municípios e distritos analisados (linha negra). Os pontos negros na Figura 1 correspondem às taxas de homicídio de negros e os pontos brancos às de homicídio da população não-negra em cada uma das 134 unidades da Região Metropolitana de São Paulo. Como pode ser visualizado na Figura 1, a taxa de homicídio de negros encontra-se invariavelmente acima e a taxa de homicídio da população não-negra encontra-se invariavelmente abaixo da linha que demarca a taxa de homicídio de cada unidade.

A taxa de homicídio por 100 mil habitantes na Região Metropolitana de São Paulo em 2000 era de 3,3 para menores de 15 anos, e 82,9 para a população com 15 e mais anos de idade analisada neste trabalho (a idade da vítima não foi informada em 233 registros de homicídio).

Para a análise do agregado das vítimas de homicídio da Região Metropolitana de São Paulo por sexo, escolaridade, idade e raça, puderam ser utilizados 8.991 homicídios $(86,0 \%$ do total das vítimas com município de residência informado e com 15 e mais anos de idade). Para garantir o tamanho da amostra populacional e sua representatividade, concomitantemente, o peso original de cada observação foi dividido pelo fator $v / \alpha$ 9,927172 (13.156.163/ 1.325.268).

De acordo com a Tabela 1, os riscos relativos de homicídio para pessoas com 15 e mais anos de idade, em relação às respectivas categorias de referência, eram 15,9 para sexo masculino, 4,4 para pessoas com até sete anos de estudo, 3,2 para pessoas de 15 a 34 anos de idade e 1,7 para a população negra na Região Metropolitana de São Paulo em 2000. Todos os riscos relativos na análise univariada resultaram ser estatisticamente significativos - os intervalos de confiança (IC95\%) figuram na Tabela 1.

A Tabela 2, elaborada com base na análise descritiva, apresenta as taxas de homicídio para os 16 grupos compostos pelas quatro variáveis desdobradas em duas categorias. As taxas de homicídio mais altas foram registradas pelos grupos compostos por homens de baixa escolaridade e jovens (respectivamente não-negros e negros); seguidos pelos grupos compostos por homens de baixa escolaridade com 35 e mais anos de idade (não-negros e negros). Os grupos compostos por homens de baixa escolaridade e jovens, que representavam 9,6\% (5,0\% + $4,6 \%)$ da população, somavam $58,1 \%(30,1 \%+$ 28,0\%) dos homicídios na Região Metropolitana de São Paulo em 2000.

A Tabela 3, elaborada baseando-se na análise multivariada de regressão logística, apresenta os dois modelos descritos em Métodos com as respectivas odds ratio e intervalos de confiança. No Modelo 1, tanto a variável escolaridade como a variável raça são estatisticamente significativas. No Modelo 2, com a introdução das variáveis demográficas sexo e idade, a variável raça deixa de ser significativa. No Modelo 2, as odds ratio resultaram ser 14,484

Taxa de homicídio e risco relativo (15 e mais anos de idade). Região Metropolitana de São Paulo, Brasil, 2000.

\begin{tabular}{|c|c|c|c|c|}
\hline Variável/categoria & Homicídio (n) & População (x 1.000) & Taxa (por 100.000) & Risco relativo (IC95\%) \\
\hline \multicolumn{5}{|l|}{ Sexo } \\
\hline Feminino & 587 & 6.914 & 8,5 & 1,0 \\
\hline Masculino & 8.404 & 6.242 & 134,6 & $15,9(13,0-19,3)$ \\
\hline \multicolumn{5}{|l|}{ Anos de estudo } \\
\hline $8 \mathrm{e}+$ & 1.887 & 7.063 & 26,7 & 1,0 \\
\hline Até 7 & 7.104 & 6.093 & 116,6 & $4,4(3,8-5,1)$ \\
\hline \multicolumn{5}{|l|}{ Idade (anos) } \\
\hline $35 e+$ & 2.114 & 6.504 & 32,5 & 1,0 \\
\hline $15-34$ & 6.877 & 6.652 & 103,4 & $3,2(2,8-3,7)$ \\
\hline \multicolumn{5}{|l|}{ Raça } \\
\hline Não-negra & 5.109 & 9.043 & 56,5 & 1,0 \\
\hline Negra & 3.882 & 4.113 & 94,4 & $1,7(1,5-1,9)$ \\
\hline Total & 8.991 & 13.156 & 68,3 & \\
\hline
\end{tabular}

Fonte: Fundação Seade 16 e IBGE 18. 


\section{Tabela 2}

Taxa de homicídio em 16 grupos (15 e mais anos de idade).

Região Metropolitana de São Paulo, Brasil, 2000.

\begin{tabular}{|c|c|c|c|c|c|}
\hline \multirow[t]{2}{*}{ Grupo } & \multicolumn{2}{|c|}{ Homicídio } & \multicolumn{2}{|c|}{ População } & \multirow{2}{*}{$\begin{array}{l}\text { Taxa (por } \\
100.000)\end{array}$} \\
\hline & $\mathrm{n}$ & $\%$ & $\mathrm{n}$ & $\%$ & \\
\hline \multicolumn{6}{|l|}{ Sexo feminino } \\
\hline \multicolumn{6}{|l|}{$8+$ anos de estudo } \\
\hline \multicolumn{6}{|l|}{$35+$ anos de idade } \\
\hline Raça não-negra & 50 & 0,6 & 1.196 & 9,1 & 4,2 \\
\hline Raça negra & 14 & 0,2 & 240 & 1,8 & 5,8 \\
\hline \multicolumn{6}{|c|}{ Até 35 anos de idade } \\
\hline Raça não-negra & 90 & 1,0 & 1.663 & 12,6 & 5,4 \\
\hline Raça negra & 29 & 0,3 & 619 & 4,7 & 4,7 \\
\hline \multicolumn{6}{|c|}{ Até 7 anos de estudo } \\
\hline \multicolumn{6}{|c|}{$35+$ anos de idade } \\
\hline Raça não-negra & 88 & 1,0 & 1.382 & 10,5 & 6,4 \\
\hline Raça negra & 44 & 0,5 & 694 & 5,3 & 6,3 \\
\hline \multicolumn{6}{|c|}{ Até 35 anos de idade } \\
\hline Raça não-negra & 132 & 1,5 & 610 & 4,6 & 21,6 \\
\hline Raça negra & 140 & 1,6 & 511 & 3,9 & 27,4 \\
\hline \multicolumn{6}{|l|}{ Sexo masculino } \\
\hline \multicolumn{6}{|l|}{$8+$ anos de estudo } \\
\hline \multicolumn{6}{|l|}{$35+$ anos de idade } \\
\hline Raça não-negra & 342 & 3,8 & 1.114 & 8,5 & 30,7 \\
\hline Raça negra & 106 & 1,2 & 252 & 1,9 & 42,1 \\
\hline \multicolumn{6}{|c|}{ Até 35 anos de idade } \\
\hline Raça não-negra & 815 & 9,1 & 1.421 & 10,8 & 57,3 \\
\hline Raça negra & 441 & 4,9 & 558 & 4,2 & 79,0 \\
\hline \multicolumn{6}{|c|}{ Até 7 anos de estudo } \\
\hline \multicolumn{6}{|c|}{$35+$ anos de idade } \\
\hline Raça não-negra & 882 & 9,8 & 997 & 7,6 & 88,5 \\
\hline Raça negra & 588 & 6,5 & 629 & 4,8 & 93,5 \\
\hline \multicolumn{6}{|c|}{ Até 35 anos de idade } \\
\hline Raça não-negra & 2.710 & 30,1 & 659 & 5,0 & 411,0 \\
\hline Raça negra & 2.520 & 28,0 & 611 & 4,6 & 412,3 \\
\hline Total & 8.991 & 100,0 & 13.156 & 100,0 & 68,3 \\
\hline
\end{tabular}

para o sexo masculino; 5,161 para até sete anos de estudo; e 3,769 para 15 a 34 anos de idade.

Na distribuição da população com 15 e mais anos de idade na Região Metropolitana de São Paulo em 2000 por raça, os negros estavam sobre-representados em todas as três demais categorias de exposição (Tabela 4). Os negros representavam $31,3 \%$ da população com 15 e mais anos de idade na Região Metropolitana de São Paulo em 2000, mas correspondiam a 32,8\% dos homens, $40,1 \%$ do segmento com até sete anos de estudo e $34,6 \%$ da população de 15 a 34 anos de idade. Em relação à participação do negro na população com 15 e mais anos de idade, a sobreposição do negro entre pessoas com até sete anos de estudo era de $28,0 \%$; entre homens $5,0 \%$; e entre pessoas de 15 a 34 anos de idade $11,0 \%$.

\section{Discussão}

As atuais taxas de homicídio do Brasil destacam-se internacionalmente e as da Região Metropolitana de São Paulo evidenciam-se entre as taxas de homicídio das regiões metropolitanas do país. Drumond 7 aponta que as vítimas fatais da violência em São Paulo são predominantemente pessoas do sexo masculino, de baixa escolaridade, jovens e negros. Hannon et al. 10 , em estudo realizado para 134 cidades norteamericanas, concluem que a variável raça não é estatisticamente significativa para a taxa de homicídio, quando devidamente controlados os coeficientes de pobreza. Na Região Metropolitana de São Paulo em 2000, de acordo com a presente pesquisa, o risco relativo de homicídio para negros em relação a não-negros $(1,7)$ reduz-se para 1,3 , mas continua sendo estatisticamente significativo quando ajustado pela variável escolaridade como uma aproximação do rendimento das vítimas. A variável raça, contudo, deixa de ser estatisticamente significativa quando se controlam, além da escolaridade, as variáveis demográficas sexo e idade da vítima. Os resultados do presente estudo indicam que a probabilidade de uma pessoa jovem do sexo masculino com baixa escolaridade ser assassinada é a mesma para negros e não-negros.

Como vimos em Métodos, a raça/cor da população é autodenominada pelo entrevistado e a da vítima de homicídio é atribuída pelo médico legista. Mas, de acordo com Teixeira 21, que confrontou o critério de autodenominação com o critério de atribuição da identidade racial, entrevistados e entrevistadores, com base na aparência, compartilham de um mesmo código para a identificação étnica. Como disse uma das pessoas entrevistadas "eu me identifico como negra porque é assim que eu sou percebida socialmente". Embora Telles 22 considere que a classificação racial brasileira é em grande parte ambígua, na Pesquisa Datafolha de 1995, em que seu trabalho é baseado, $88,0 \%$ das pessoas foram consistentemente classificadas como brancas ou negras (pardos e pretos) tanto pelo entrevistador como pelo entrevistado.

A sobre-representação de negros entre as vítimas não está relacionada à variável raça, mas sim às demais variáveis analisadas neste estudo. A sobre-representação dos negros entre as vítimas de homicídio decorre de sua sobre-re- 
presentação tanto entre pessoas de baixa escolaridade (e renda) como entre jovens e pessoas do sexo masculino.

Como o numerador da taxa de homicídio é essencialmente composto por homens com baixa escolaridade e jovens (principal grande grupo de exposição) e o denominador para negros é composto por relativamente menor número de pessoas do sexo feminino, com 8 e mais anos de estudo e com 35 e mais anos de idade, a taxa bruta de homicídio para negros resulta ser relativamente maior que a de não-negros. Quando tanto o numerador quanto o denominador restringem-se ao grande grupo de exposição, as taxas de homicídio de negros e nãonegros passam a ser iguais.

Assim, podemos concluir que, embora os homicídios na Região Metropolitana de São Paulo em 2000 tenham atingido essencialmente pessoas do sexo masculino, de baixa escolaridade, jovens e negros, a característica negro reflete a sobre-representação dos negros entre homens, pobres e jovens. Homens com baixa escolaridade e jovens, negros e não-negros, apresentaram a mesma probabilidade de serem assassinados.

De acordo com Beato Filho et al. 23, os homicídios ocorrem em regiões assoladas pelo tráfico de drogas e, de acordo com Zaluar 4, as vítimas fatais da violência não são pessoas estranhas ao crime, mas os próprios jovens pobres aliciados pela droga que se exterminam mutuamente na lógica do tráfico. $\mathrm{O}$ tráfico de drogas é responsável por um número vultoso de homicídios que têm como origem: (a) o código penal cáustico instituído pelo tráfico que, nos bairros pobres, tem levado seus jovens integrantes à morte; (b) a concorrência sangüinária entre traficantes derivada dos lucros extraordinários desse comércio ilícito; e (c) o confronto com a polícia, em alguns casos com po- liciais corruptos que exigem sua parte nos lucros. Analisada desta forma, a sobre-representação de negros entre homens, pobres e jovens é responsável não só pela sobre-representação de negros entre as vítimas fatais da violência como pela sobre-representação de negros entre os agressores.

Cidadãos negros aparecem no imaginário coletivo como potenciais criminosos, suspeitos por excelência 24 . O médico legista baiano Nina Rodrigues, na passagem do século XIX para o XX, considerava que os negros eram criminosos em decorrência de seu estado de evolução jurídica e de suas crenças religiosas 25 . Mesmo os negros não estão imunes ao preconceito contra o negro 26 , ou seja, o preconceito pode ser interiorizado por pessoas do próprio grupo discriminado, com impactos sobre a auto-estima: “...soldados, quase todos pretos, dando porrada na nuca de malandros pretos" (Hai$t i$, de Gilberto Gil \& Caetano Veloso).

\begin{tabular}{|c|c|c|}
\hline \multicolumn{3}{|c|}{$\begin{array}{l}\text { Regressão logística para homicídio (15 e mais anos de idade). } \\
\text { Região Metropolitana de São Paulo, Brasil, } 2000 .\end{array}$} \\
\hline Variável & Odds ratio & IC95\% \\
\hline \multicolumn{3}{|l|}{ Modelo 1} \\
\hline Raça & 1,320 & $1,156-1,508$ \\
\hline Anos de estudo & 4,159 & $3,538-4,889$ \\
\hline \multicolumn{3}{|l|}{ Modelo 2} \\
\hline Raça & 1,073 & $0,939-1,227$ \\
\hline Anos de estudo & 5,161 & $4,384-6,075$ \\
\hline Sexo & 14,484 & $11,125-18,858$ \\
\hline Idade & 3,769 & $3,228-4,401$ \\
\hline
\end{tabular}

Tabela 4

Distribuição da população por raça (15 e mais anos de estudo). Região Metropolitana de São Paulo, Brasil, 2000.

\begin{tabular}{cccccccc}
\hline Categoria de exposição & \multicolumn{3}{c}{$\begin{array}{c}\text { Número } \\
\text { Negra }\end{array}$} & Não-negra & Total & & \multicolumn{2}{c}{$\begin{array}{c}\text { Participação (\%) } \\
\text { Negra }\end{array}$} & Não-negra & Total & Sobre- \\
representação*
\end{tabular}

Fonte: Fundação Seade 16, IBGE 18 .

* Sobre-representação de negros nas categorias em relação à participação de negros na população (31,3\%). 
Em 1996, os culpados por uma chacina ocorrida em um bar de classe média na cidade de São Paulo, todos pretos, foram prontamente encontrados pela polícia. Cerca de dez dias depois, a polícia liberou todos os supostos culpados e apresentou os novos suspeitos do crime, todos brancos 12. Esta postura também explica porque réus negros têm maior probabilidade de serem punidos, comparativamente a réus brancos que cometeram crimes de idêntica natureza 24 .

Em relação ao preconceito contra os negros no país, deve-se considerar que a discriminação racial sem tensões e inquietação social restringem as oportunidades econômicas, educacionais, sociais e políticas dos negros, o que contribui para perpetuar o passado no presente e impede a existência e o surgimento de uma verdadeira democracia racial no Brasil 13. A harmonia racial tem sido utilizada pelas elites do país para enaltecer a sociedade multirracial brasileira. O mito da democracia racial brasi- leira legitima a desigualdade e impede que a situação real se transforme numa questão pública. Quanto mais longe se está do fim do sistema escravista, menor o poder explicativo da escravidão e da origem social dos negros como causa de sua subordinação social corrente, e maior o poder explicativo do racismo e da discriminação contemporâneos. As oportunidades dos filhos negros são menores que a dos não-negros mesmo quando se controla a posição social das famílias de origem 14 .

As políticas de ação afirmativa, de caráter temporário e que estão sendo tardiamente implementadas hoje no país, visam a compensar a população afro-descendente pelos obstáculos que enfrentaram e continuam enfrentando na afirmação de sua plena cidadania. Neste sentido, vale lembrar, o importante não é simplesmente a igualdade de direitos, mas sim a igualdade de oportunidades de acesso à educação e ao emprego 28.

\section{Resumo}

O trabalho analisa a taxa de homicídio na Região Metropolitana de São Paulo, Brasil, por raça, controladas as variáveis de ordem sócio-econômica (escolaridade) e demográfica (sexo e idade da vítima). Com base nas estatísticas vitais da Fundação Seade e do Censo Demográfico do IBGE, foram calculadas as taxas de homicídio em 2000 para a raça negra e para a população não-negra, em 134 unidades da Região Metropolitana de São Paulo (38 municípios e 96 distritos da capital). Para o agregado da mesma região, foram construídas duas categorias (de referência e de exposição) para as variáveis raça, escolaridade, sexo e idade. A pesquisa utiliza análise descritiva e regressão logística múltipla. Embora a taxa bruta de homicídio de negros fosse invariavelmente superior à taxa para não-negros, a variável raça resultou ser não significativa quando controladas as variáveis anos de estudo, sexo e idade. A taxa de homicídio calculada para negros na Região Metropolitana de São Paulo em 2000 é maior que a taxa calculada para não-negros, em decorrência da sobre-representação de negros tanto na população com baixa escolaridade como na população masculina e jovem, principais categorias de exposição para homicídios.

Homicídio; Coeficiente de Mortalidade; Causa da Morte; Negros

\section{Colaboradores}

Todos os autores tiveram a mesma participação, contribuindo em todas as etapas da pesquisa (levantamento bibliográfico, dados estatísticos, análise estatística e redação).

\section{Agradecimentos}

Aos professores César Roberto Leite da Silva, da Pontifícia Universidade Católica de São Paulo e Fernando Garcia, da Fundação Getúlio Vargas de São Paulo, pela assessoria na análise estatística; e à Luciana Zilles Lima, do Laboratório de Economia Social, pela colaboração. Trabalho realizado com o apoio do Conselho de Ensino e Pesquisa, Pontifícia Universidade Católica de São Paulo e do Conselho Nacional de Desenvolvimento Científico e Tecnológico (CNPq), apresentado no VI Congresso Brasileiro de Epidemiologia em Recife, 2004 e no XIV Encontro Nacional de Estudos Populacionais em Caxambu, 2004. 


\section{Referências}

1. Jorge MHPM, Gawryszewski VP, Latorre MRDO. Análise dos dados de mortalidade. Rev Saúde Pública 1997; 31 (4 Suppl):5-25.

2. Rede Interagencial de Informações para a Saúde. Indicadores e dados básicos para a saúde, Brasil, 2003. http://tabnet.datasus.gov.br/cgi/tabcgi.exe ?idb2003/c09.def (acessado em 24/Fev/2005).

3. United Nations. Demographic yearbook 2000. http: //unstats.un.org/unsd/demographic/products/d yb/DYB2000/Table21.pdf (acessado em 25/Fev/ 2005).

4. Zaluar A. Da revolta ao crime S.A. São Paulo: Moderna; 1996.

5. Levitt SD. Juvenile crime and punishment. J Polit Econ 1998; 106:1156-85.

6. Dobrin A. The risk of offending on homicide victimization: a case control study. Journal of Research in Crime and Delinquency 2001; 38:154-73.

7. Drumond Jr. M. Vida e morte em São Paulo. São Paulo: Brasiliense; 2002.

8. Batista LE, Escuder MML, Pereira JCR. A cor da morte: causas de óbito segundo características de raça no Estado de São Paulo, 1999 a 2001. Rev Saúde Pública 2004; 38:630-6.

9. Ousey GC, Lee MR. Investigating the connections between race, illicit drug markets, and lethal violence, 1984-1997. Journal of Research in Crime and Delinquency 2004; 41:352-83.

10. Hannon L, Knapp P, DeFina R. Racial similarity in the relationship between poverty and homicide rates: comparing retransformed coefficients. http://www88.homepage.villanova.edu/lance.ha nnon/Retransformed\%20Coefficients\%20and\%2 0Race-specific\%20Homicide.pdf (acessado em 02/Fev/2005).

11. Fernandes F. O negro no mundo dos brancos. São Paulo: Difusão Européia do Livro; 1972.

12. Schwarcz LM. Nem preto nem branco, muito pelo contrário, cor e raça na intimidade. In: Schwarcz LM, organizador. História da vida privada no Brasil: contrastes da intimidade contemporânea. v. 4. São Paulo: Companhia das Letras; 1998. p. 175-243.

13. Henriques R. Desigualdade racial no Brasil: evolução das condições de vida na década de 90 . Textos para Discussão 807. http://www.ipea.gov.br (acessado em 29/Ago/2004).

14. Hasenbalg CA. Discriminação e desigualdades raciais no Brasil. Rio de Janeiro: Graal; 1979.

15. Silva NV. O preço da cor: diferenciais raciais na distribuição da renda no Brasil. Pesqui Planej Econ 1980; 10:21-41.
16. Fundação Seade. Sistema de estatísticas vitais (microdados) [CD-ROM]. São Paulo: Fundação Seade; 2002.

17. Programa de Aprimoramento de Dados sobre a Mortalidade no Município de São Paulo. Sistema de informação sobre mortalidade (banco de dados) [CD-ROM]. São Paulo: Programa de Aprimoramento de Dados sobre a Mortalidade no $\mathrm{Mu}$ nicípio de São Paulo; 2003.

18. Instituto Brasileiro de Geografia e Estatística. Censo demográfico 2000, resultados da amostra, agregado de áreas de ponderação (microdados) [CD-ROM]. Rio de Janeiro: Instituto Brasileiro de Geografia e Estatística; 2002.

19. Jacooud L, Beghin N. Desigualdades raciais no Brasil. Brasília: Instituto de Pesquisa Econômica Aplicada; 2002.

20. Ramos L, Vieira ML. A relação entre educação e salários no Brasil. In: Instituto de Pesquisa Econômica Aplicada, organizador. Economia brasileira em perspectiva. v. 2. Rio de Janeiro: Instituto de Pesquisa Econômica Aplicada; 1996. p. 493-510.

21. Teixeira MP. Negros na universidade: identidade e trajetórias de ascensão social no Rio de Janeiro. Rio de Janeiro: Pallas; 2003.

22. Telles E. Racismo à brasileira. Rio de Janeiro: Relume Dumará; 2003.

23. Beato Filho CC, Assunção RM, Silva BFA, Marinho FC, Reis IA, Almeida MCM. Conglomerados de homicídios e o tráfico de drogas em Belo Horizonte, Minas Gerais, Brasil, de 1995 a 1999. Cad Saúde Pública 2001; 17:1163-71.

24. Adorno S. Discriminação racial e justiça criminal em São Paulo. Novos Estudos CEBRAP 1995; 43: 45-63.

25. Rodrigues N. Os africanos no Brasil. São Paulo: Companhia Editora Nacional; 1935.

26. Fausto B. Crime e cotidiano: a criminalidade em São Paulo (1880-1924). São Paulo: Brasiliense; 1984.

27. Teixeira MP, Siss A, Muller MLR, Oliveira I, Brandão AAP. Projeto políticas de ação afirmativa nas universidades Federal Fluminense e Federal do Mato Grosso. In: Anais do 13o Encontro Nacional de Estudos Populacionais. http://www.abep.org. br (acessado em 29/Ago/2004).

Submetido em 18/Out/2004

Versão final reapresentada em 04/Mar/2005

Aprovado em 15/Abr/2005 